\title{
Puerarin suppresses cell growth and migration in HPV-positive cervical cancer cells by inhibiting the PI3K/mTOR signaling pathway
}

\author{
LIHUA JIA $^{1}$, YULING HU ${ }^{2}$, GUOHUA YANG ${ }^{2}$ and PEILING LI ${ }^{1}$ \\ ${ }^{1}$ Department of Obstetrics and Gynecology, The Second Affiliated Hospital of Harbin Medical University, \\ Harbin, Heilongjiang 150086; ${ }^{2}$ Department of Obstetrics and Gynecology, Tongzhou Maternal \\ and Child Health Hospital of Beijing, Beijing 101101, P.R. China
}

Received November 29, 2017; Accepted June 1, 2018

DOI: $10.3892 /$ etm.2019.7589

\begin{abstract}
Puerarin is an effective component that is present in high concentrations in the Pueraria lobata plant and is extensively distributed throughout nature. Puerarin possesses a number of pharmacological effects and has strong pharmacological activity with few side effects and extensive clinical applications. The aim of the present study was to explore the effects of Puerarin on the apoptosis of human papillomavirus (HPV)-positive cervical cancer cells and the underlying molecular mechanisms. MTT assay, lactate dehydrogenase activity and Annexin V/fluorescein isothiocyanate/propidium iodide analysis were used to analyze cell growth of HPV-positive HeLa cervical cancer cells treated with Puerarin. Western blotting was performed to measure protein expression in the treated cells. Puerarin significantly reduced cell proliferation and induced apoptosis in HeLa cells. In addition, it was observed that Puerarin significantly enhanced caspase-3/9 activities and significantly increased B-cell lymphoma 2-asscoiate $\mathrm{X}$ protein expression in HeLa cells. Puerarin suppressed phosphatidylinositol-3 kinase (PI3K), phosphorylated (p)-protein kinase B (Akt) and p-mammalian target of rapamycin (mTOR) protein expression in HeLa cells. These results indicate that Puerarin induces apoptosis in HPV-positive HeLa cervical cancer cells via inhibiting PI3K/Akt/mTOR signaling.
\end{abstract}

\section{Introduction}

Cervical cancer is the second most common type of malignant tumor in females worldwide and the most common in a number

Correspondence to: Mr. Peiling Li, Department of Obstetrics and Gynecology, The Second Affiliated Hospital of Harbin Medical University, 148 Care Road, Harbin, Heilongjiang 150086, P.R. China E-mail: iikuvama2302@126.com

Key words: Puerarin, human papillomavirus, cervical cancer cell, phosphatidylinositol-3 kinase, protein kinase B, mammalian target of rapamycin of developing countries, representing a large socioeconomic burden (1). The International Agency for Research on Cancer reported that $\sim 300,000$ mortalities are attributed to cervical cancer annually in China $(2,3)$.

Human papillomavirus (HPV)-18 is regarded as an important strain of HPV that causes precancerous lesions, which subsequently develop into cervical cancer and is associated with $>90 \%$ of all cervical cancer cases (4). The prevalence of HPV-18 infection in women continues to increase globally; however, in the majority of cases infection does not progress to the disease stage (4). It is therefore important to determine factors aside from infection that lead to carcinogenesis and the progression of cervical cancer (5).

Recent studies have revealed that the phosphatidylinositol-3 kinase $(\mathrm{PI} 3 \mathrm{~K})$ /protein kinase $\mathrm{B}(\mathrm{Akt})$ signal transduction pathway (6) serves a key role in a number of cellular activities, including apoptosis, aging and proliferation (7). The PI3K/Akt signal transduction pathway responds to various intracellular and extracellular survival pressures to adaptively regulate these processes (7). A previous study demonstrated that ionizing radiation, ultraviolet rays and cytotoxic drugs may activate the PI3K/Akt signal transduction pathway (8).

Mammalian target of rapamycin (mTOR) is a type of serine/threonine protein kinase that is highly evolutionarily conserved and is observed in a wide variety of organisms (9). The mTOR signaling pathway serves an important role in the growth and proliferation of normal cells, however it is also closely associated with the growth, proliferation, differentiation, apoptosis and metabolism of a number of types of malignant tumor (10). mTOR expression has been reported to be upregulated in multiple tumors, including breast cancer, colon cancer and lymphoma (10).

Puerarin is an isoflavonoid monomer that may be isolated and extracted from the leguminous plant Pueraria lobate (11). Puerarin has strong pharmacological activity (12) and a wide range of pharmacological effects, including anti-arrhythmia, anti-myocardial infarction, anti-angiectasis, antitumor, microcirculation improvement, blood fat reduction, increased cerebral blood flow, protection against oxidation, regulation of bone metabolism and reduction of intraocular pressure $(11,12)$. Huang et al (13) demonstrated that Puerarin induces cell 
apoptosis in human chondrosarcoma cells via inhibiting the PI3K/Akt signaling pathway. The aim of the present study was to explore the effects of Puerarin on apoptosis in HPV-positive cervical cancer cells and the molecular mechanisms responsible.

\section{Materials and methods}

Cell culture. A HeLa HPV-18-positive cervical cancer cell line was obtained from the American Type Culture Collection (cat. no. CRM-CCL-2; Manassas, VA, USA) and cultured in Dulbecco's modified Eagle's medium (DMEM) supplemented with $10 \%$ fetal bovine serum (FBS; both Gibco; Thermo Fisher Scientific, Inc., Waltham, MA, USA), 2 mM/L-glutamine, $100 \mathrm{U} / \mathrm{ml}$ penicillin and $100 \mathrm{mg} / \mathrm{ml}$ streptomycin (both Sigma-Aldrich; Merck KGaA, Darmstadt, Germany) at $37^{\circ} \mathrm{C}$ in a humidified atmosphere containing $5 \% \mathrm{CO}_{2}$.

MTT assay and lactate dehydrogenase (LDH) activity. HeLa cells were seeded at a density of $5 \times 10^{3}$ cells/well in 96 -well plates and incubated overnight at $37^{\circ} \mathrm{C}$. The cells were treated with $0,0.25,0.50,1.00$ or $2.00 \mathrm{mM}$ Puerarin (Sigma-Aldrich; Merck $\mathrm{KGaA}$ ) for 24,48 and $72 \mathrm{~h}$ at $37^{\circ} \mathrm{C}$ as previously described (14). A total of $20 \mu \mathrm{l}$ MTT solution (Sigma-Aldrich; Merck KGaA) was added to each well and the cells were incubated for a further $4 \mathrm{~h}$. A total of $150 \mu \mathrm{l}$ dimethyl sulfoxide was added to each well to dissolve the purple formazan and the absorption was measured using a microplate reader (BioTek Instruments, Inc., Winooski, VT, USA) at $492 \mathrm{~nm}$.

HeLa cells were seeded in 6-well plates at a density of $5 \times 10^{6}$ cells/well and were treated with $0.50,1.00$ or $2.00 \mathrm{mM}$ Puerarin for $48 \mathrm{~h}$ at $37^{\circ} \mathrm{C}$. The LDH activity level was subsequently measured using an LDH activity kit (C0016; Beyotime Institute of Biotechnology, Haimen, China) and the absorption was measured with a microplate reader at $450 \mathrm{~nm}$.

Annexin V/fluorescein isothiocyanate/propidium iodide (PI) analysis. HeLa cells were seeded in 6-well plates overnight $\left(5 \times 10^{6}\right.$ cells/well $)$ at $37^{\circ} \mathrm{C}$. The cells were treated with $0.50,1.00$ or $2.00 \mathrm{mM}$ Puerarin for $48 \mathrm{~h}$ at $37^{\circ} \mathrm{C}$, fixed with $4 \%$ paraformaldehyde for $15 \mathrm{~min}$ at room temperature, stained with Annexin V/phycoerythrin and PI (Sigma-Aldrich; Merck $\mathrm{KGaA}$ ) at room temperature for $15 \mathrm{~min}$ and analyzed using flow cytometry (FACScan; BD Biosciences, San Jose, CA, USA). Data were analyzed using FlowJo 7.6.1 (FlowJo LLC, Ashland, OR, USA).

Migration assay. HeLa cells $\left(1 \times 10^{5}\right)$ were seeded in the upper chamber of 24-well plates with Transwell inserts (pore size, $8 \mu \mathrm{m}$; Millipore; Merck KGaA) containing DMEM. The lower chamber contained DMEM supplemented with 10\% FBS. Following $48 \mathrm{~h}$, cells from the lower surface of the inserts were fixed with $4 \%$ paraformaldehyde for $10 \mathrm{~min}$ at room temperature and stained with $1 \%$ crystal violet for $30 \mathrm{~min}$ at room temperature. Migrating cells were visualized using a light microscope (magnification, x100).

Measuring caspase-3/9 activity and a DAPI assay. Total protein was extracted from cells using a radioimmunoprecipitation buffer (Sigma-Aldrich; Merck KGaA). A total of $10 \mu \mathrm{g}$ protein was incubated with caspase- 3 and caspase- 9 activity kits (C1115 and C1158; Beyotime Institute of Biotechnology) for $2 \mathrm{~h}$ at $37^{\circ} \mathrm{C}$, according to the manufacturer's instructions. The absorption was measured using a plate reader at $405 \mathrm{~nm}$.

HeLa cells $\left(5 \times 10^{6}\right.$ cells/well $)$ were seeded in 6 -well plates and incubated overnight at $37^{\circ} \mathrm{C}$. The cells were then treated with $0.50,1.00$ or $2.00 \mathrm{mM}$ Puerarin for $48 \mathrm{~h}$ at $37^{\circ} \mathrm{C}$, washed with PBS, fixed with $4 \%$ paraformaldehyde for $15 \mathrm{~min}$ at room temperature and stained with DAPI $(5 \mathrm{mg} / \mathrm{ml})$ for $30 \mathrm{~min}$ in darkness at room temperature. Cells were observed using a fluorescence microscope (magnification, x100).

Western blotting. Total protein was extracted from the cells as described above. The proteins $(50 \mu \mathrm{g})$ were separated using 10\% SDS-PAGE gels and transferred to nitrocellulose membranes. The membranes were blocked with 5\% non-fat milk in TBST for $1 \mathrm{~h}$ at $37^{\circ} \mathrm{C}$ and incubated with primary antibodies against Bax (sc-6236; 1:10,000), PI3K (sc-293172; 1:2,000), phosphorylated (p)-Akt (sc-7985-R; 1:1,000), Akt (sc-135829; 1:1,000), p-mTOR (sc-293133; 1:1,000), mTOR (sc-1549; 1:1,000), and GADPH (sc-47724; 1:1,000; all Santa Cruz Biotechnology, Inc., Dallas, TX, USA) overnight at $4^{\circ} \mathrm{C}$. The membranes were subsequently incubated with goat anti-rabbit IgG-horseradish peroxidase secondary antibodies (sc-2004; 1:5,000; Santa Cruz Biotechnology, Inc.) at $37^{\circ} \mathrm{C}$ for $1 \mathrm{~h}$ and developed using an enhanced chemiluminescent detection system (Beyotime Institute of Biotechnology). The protein bands were scanned using a Fujifilm LAS-3000 Imaging system (Fujifilm Corporation, Tokyo, Japan) and analyzed with Image Lab 3.0 (Bio-Rad Laboratories, Inc.).

Cell transfection. The PI3K plasmid was purchased from Sangon Biotech Co., Ltd. (Shanghai, China). HeLa cells were transfected with $100 \mathrm{nM}$ PI3K plasmid using Lipofectamine ${ }^{\circledR} 2000$ reagent (Invitrogen; Thermo Fisher Scientific, Inc.) following the manufacturer's protocol. At $4 \mathrm{~h}$ following transfection, medium was replaced and supplemented with $0.50,1.00$ or $2.00 \mathrm{mM}$ Puerarin and cells were cultured for $44 \mathrm{~h}$ at $37^{\circ} \mathrm{C}$.

Statistical analysis. Data are expressed as the mean \pm standard deviation and are representative of three replicates. SPSS 17.0 (SPSS, Inc., Chicago, IL, USA) was used in data analyses. Comparisons were made using one-way analysis of variance, with a Bonferroni post-hoc test. $\mathrm{P}<0.05$ was considered to indicate a statistically significant difference.

\section{Results}

Puerarin reduces cell proliferation in HeLa cells. It was investigated whether Puerarin (Fig. 1A) exerted any effects on the proliferation of HeLa cells. Puerarin inhibited the cell proliferation of HeLa cells in a dose- and time-dependent manner. Treatment with $0.5 \mathrm{mM}$ Puerarin significantly inhibited the proliferation of HeLa cells compared with the control group at $72 \mathrm{~h}$, however treatment with 1-4 mM Puerarin significantly inhibited the cell proliferation at all time points (Fig. 1B). In addition, treatment with 1 or $2 \mathrm{mM}$ Puerarin significantly increased the LDH activity (Fig. 1C) and significantly reduced the migration rate of HeLa cells compared with the control group (Fig. 1D and E). 
A

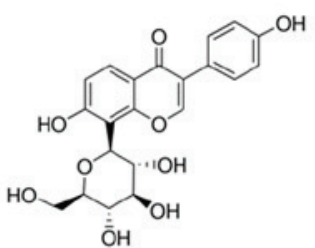

B

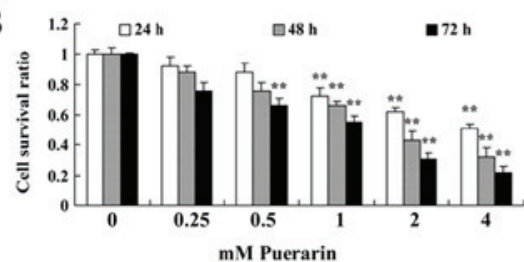

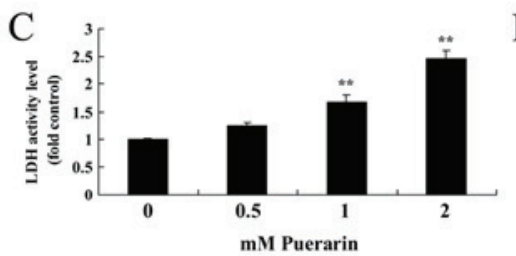

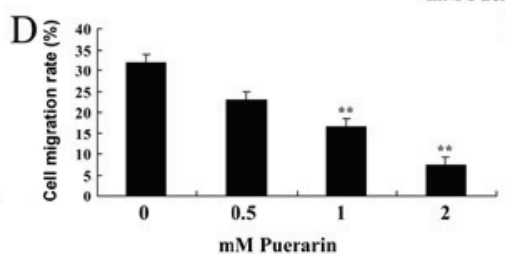

E

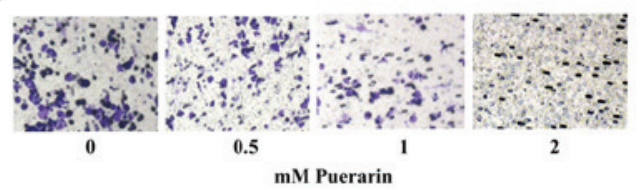

Figure 1. Puerarin reduces cell proliferation in HeLa cells. (A) Chemical structure of Puerarin. (B) Cell survival, (C) LDH activity and (D) migration rate in HeLa cells. (E) HeLa cells following treatment with $0,0.51$ or $2 \mathrm{mM}$ Puerarin for $48 \mathrm{~h}$ (magnification, $\mathrm{x} 100){ }^{* *} \mathrm{P}<0.01 \mathrm{vs}$. control group. LDH, lactate dehydrogenase.

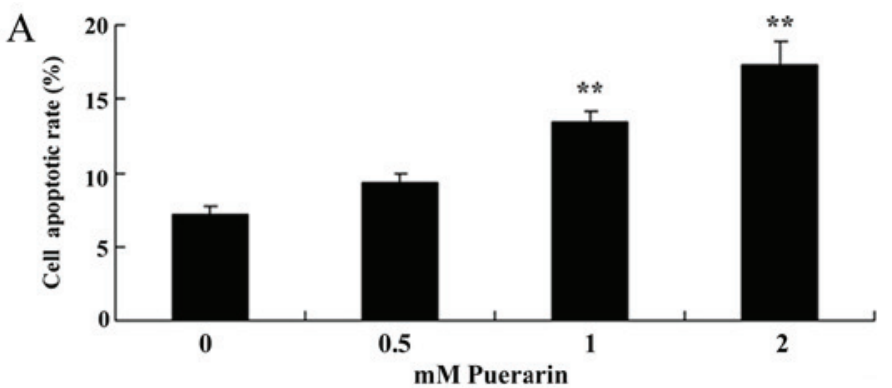

B
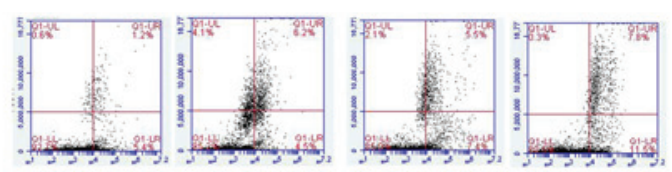

$\mathrm{C}$
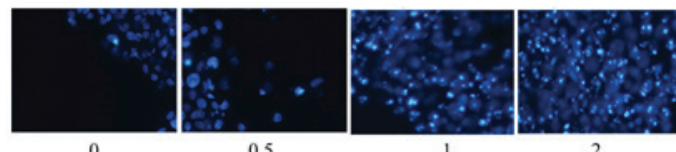

mM Puerarin
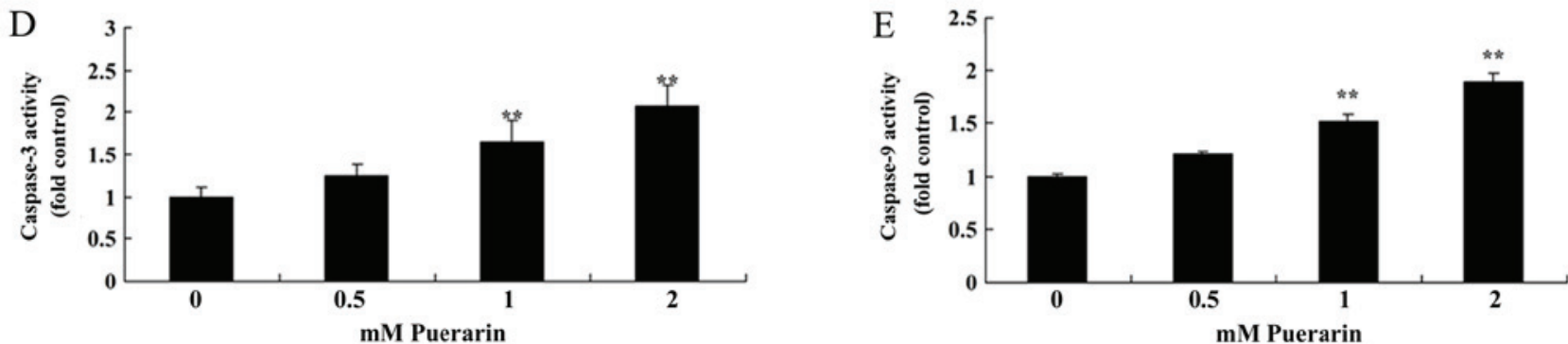

Figure 2. Puerarin induces apoptosis in HeLa cells. (A) Apoptosis rate was determined by (B) flow cytometry. (C) DAPI assay performed on HeLa cells following treatment with $0,0.51$ or $2 \mathrm{mM}$ for $48 \mathrm{~h}$ (magnification, $\mathrm{x} 100$ ). (D) Caspase-3 and (E) Caspase-9 activity. ${ }^{* *} \mathrm{P}<0.01 \mathrm{vs}$. the control group.

Puerarin induces apoptosis in HeLa cells. Flow cytometry analysis was performed to determine whether Puerarin affects the apoptosis of HeLa cells. The apoptosis rate in cells treated with Puerarin (1-2 mM) was significantly increased compared with the control group (Fig. 2A). In addition, Puerarin (1-2 mM) significantly increased Ccaspase-3 and -9 activity in HeLa cells compared with the control group (Fig. 2D and E).

Puerarin suppresses $P I 3 K, p$-Akt and $p$-mTOR protein expression in HeLa cells. The results of western blotting demonstrated that treatment with Puerarin (1-2 mM) significantly reduced PI3K (Fig. 3A), p-Akt (Fig. 3B) and p-mTOR (Fig. 3C) protein expression and significantly increased Bax (Fig. 3D) protein expression in HPV-18 positive HeLa cells compared with the control group (Fig. 3E).

An increase in PI3K reduces the anticancer effect of Puerarin in HeLa cells. The role of PI3K/p-Akt/p-mTOR signaling in the anticancer effects of Puerarin was further investigated. Transfection with the PI3K plasmid significantly increased the protein expression of PI3K (Fig. 4A), p-Akt (Fig. 4B) and p-mTOR (Fig. 4C), while Bax (Fig. 4D) expression was significantly reduced compared with the Puerarin treated group (Fig. 4E). It was also revealed that transfection with PI3K plasmids significantly ameliorated the effects of Puerarin on cell viability (Fig. 5A) and also significantly decreased the LDH activity compared with the Puerarin group (Fig. 5B). PI3K plasmid transfection significantly decreased the migration rate of HeLa cells compared with the Puerarin group (Fig. 5C and D). It was also demonstrated that PI3K transfection significantly decreased the anticancer effect of Puerarin on the rate of apoptosis and caspase-3/9 activities in HeLa cells compared with the Puerarin group (Fig. 6).

\section{Discussion}

Cervical cancer associated with HPV-infection is a potentially preventable malignancy, as a vaccination for HPV is currently available and undergoing further development. In addition, if identified at an early stage cervical cancer is readily 
A
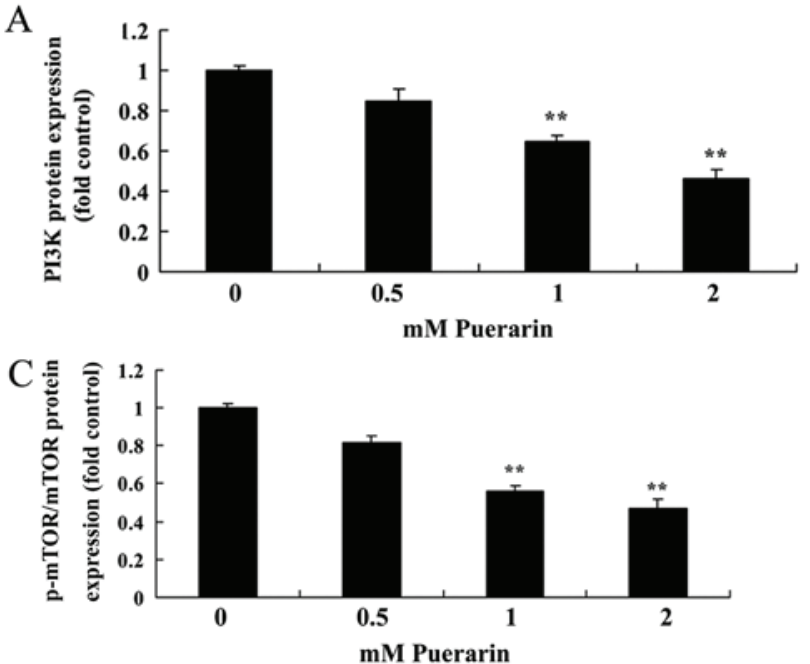

$\mathrm{D}$

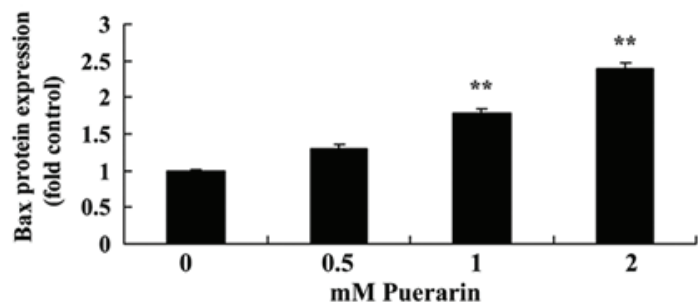

B

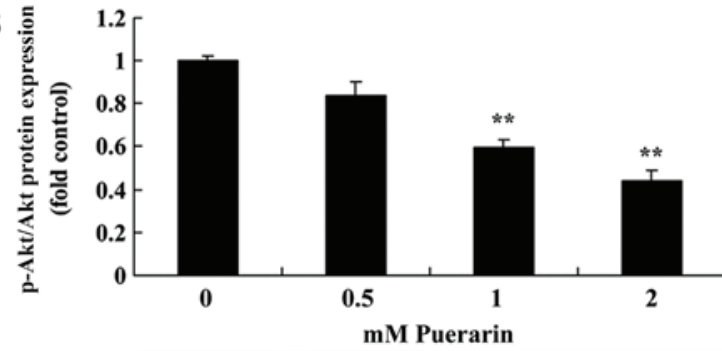

E

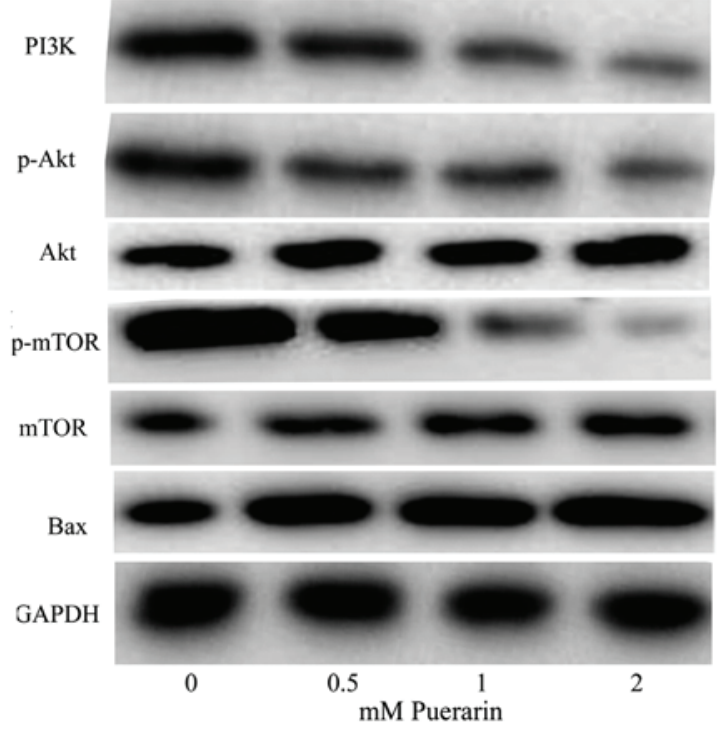

Figure 3. Puerarin suppresses PI3K, p-Akt and p-mTOR protein expression in HeLa cells. (A) PI3K, (B) p-Akt, (C) p-mTOR and (D) Bax protein expression was determined using (E) western blotting in HeLa cells. ${ }^{* *} \mathrm{P}<0.01$ vs. control group. PI3K, phosphatidylinositol-3 kinase; Akt, protein kinase B; mTOR, mammalian target of rapamycin; $p$, phosphorylated.

A

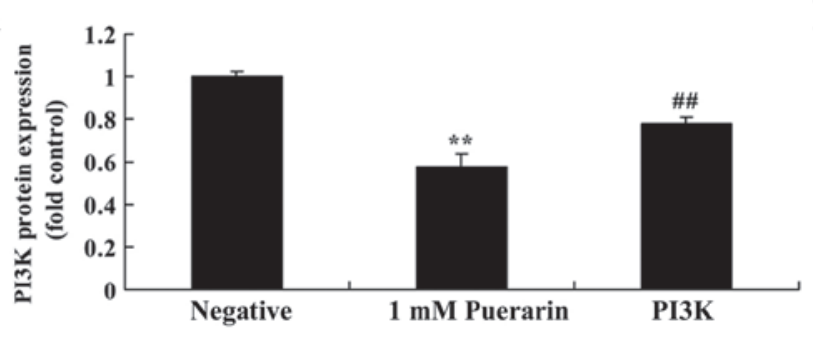

$\mathrm{C}$

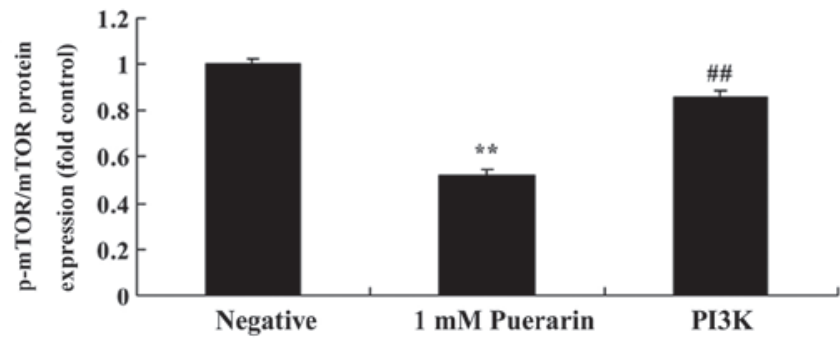

D

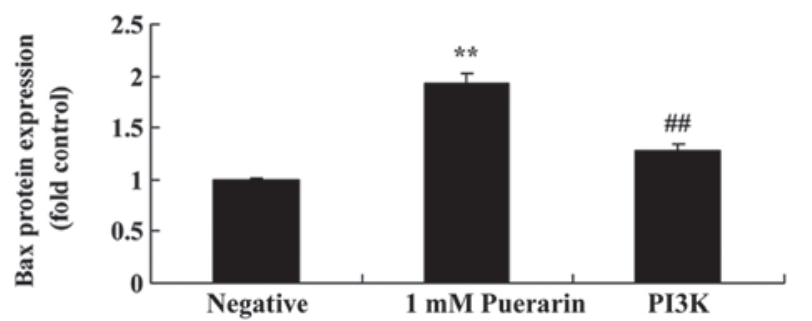

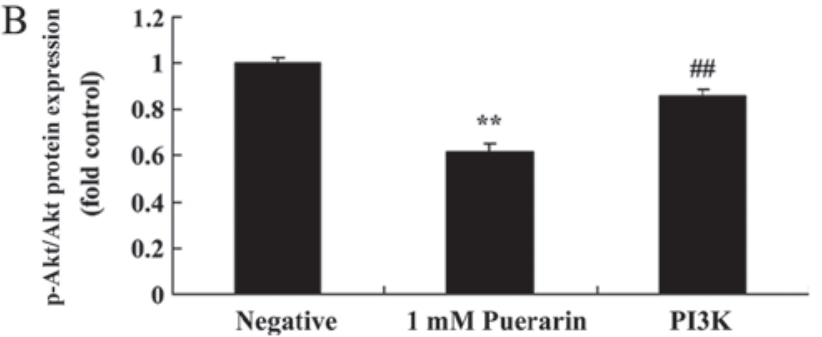

E PI3K

p-Akt

Akt
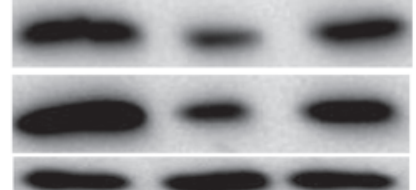

p-mTOR

mTOR

$\operatorname{Bax}$

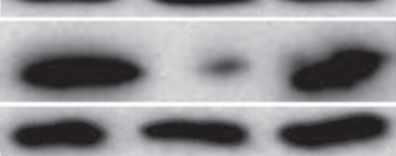

GAPDH

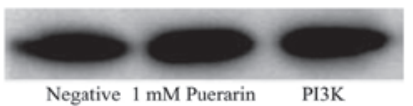

Figure 4. Transfection with PI3K plasmids reduces the anticancer effect of Puerarin in HeLa cells. (A) PI3K, (B) p-Akt, (C) p-mTOR and (D) Bax protein expression was determined using (E) western blotting in HeLa cells. ${ }^{* *} \mathrm{P}<0.01$ vs. control group. ${ }^{\# \#} \mathrm{P}<0.01 \mathrm{vs}$. $1 \mathrm{nM}$ Puerarin group. PI3K, phosphatidylinositol-3 kinase; Akt, protein kinase B; mTOR, mammalian target of rapamycin; p, phosphorylated. 

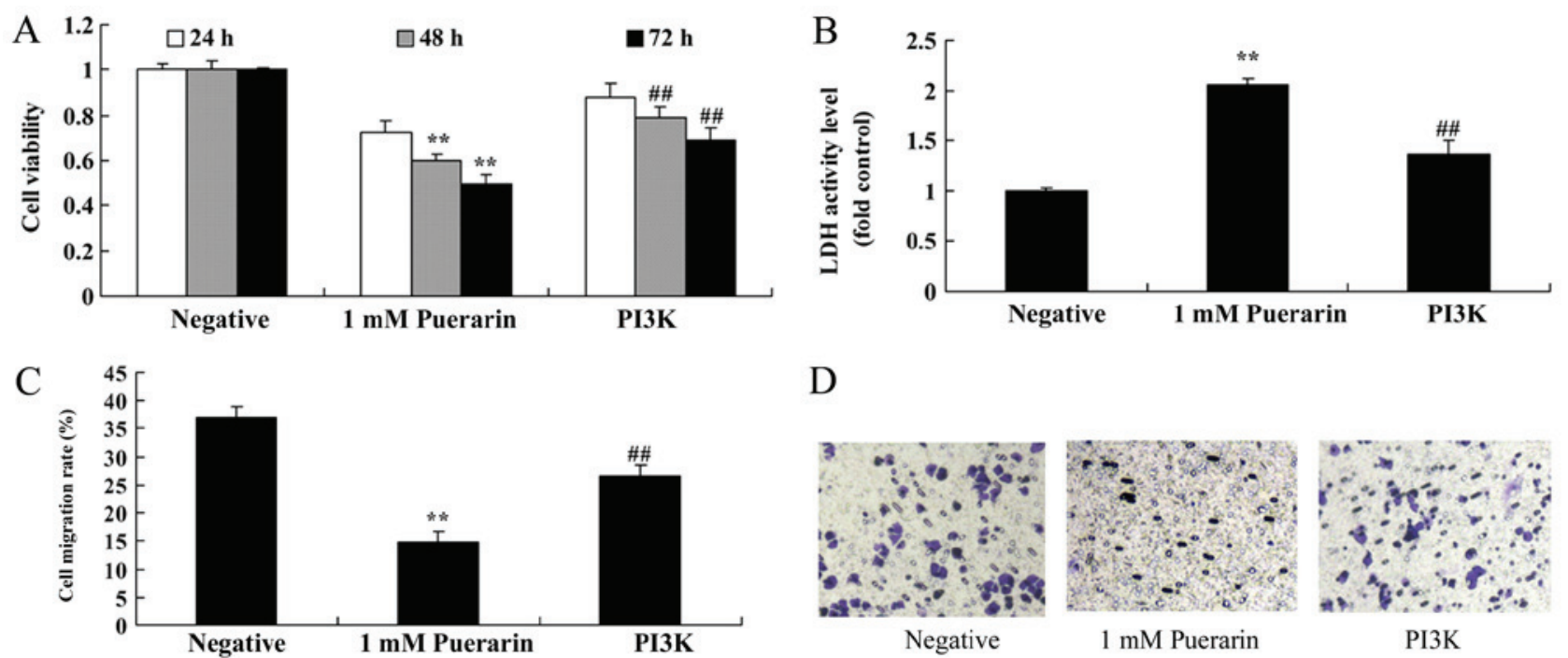

D
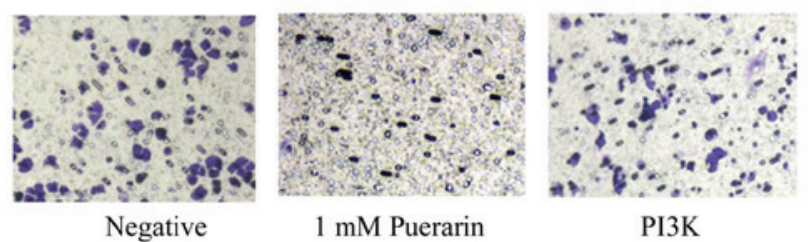

Figure 5. Transfection with PI3K plasmids reduces the effect of Puerarin on HeLa cell growth. (A) Cell viability, (B) LDH activity and (C) migration rate were assessed; (D) HeLa cells were assessed using DAPI by microscopy (magnification, $\mathrm{x} 100$ ). ${ }^{* *} \mathrm{P}<0.01$ vs. control group. ${ }^{\# \#} \mathrm{P}<0.01 \mathrm{vs} .1 \mathrm{nM}$ Puerarin group. PI3K, phosphatidylinositol-3 kinase; LDH, lactate dehydrogenase.

A
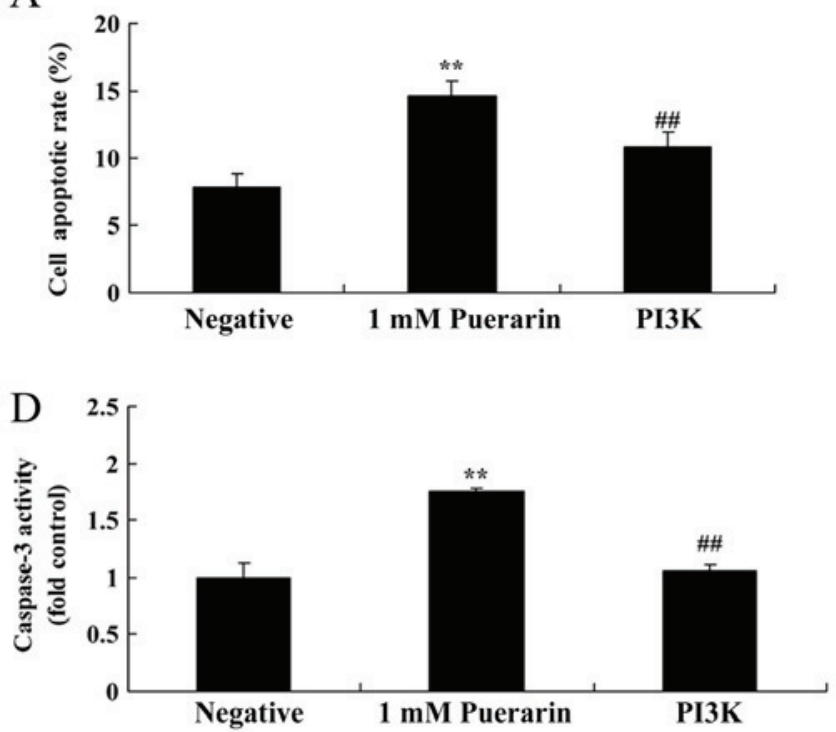

B
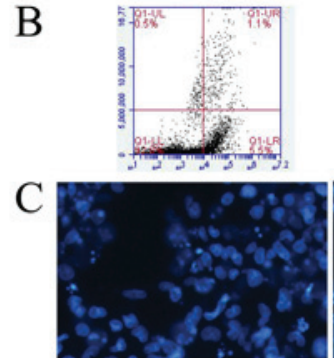

Negative

$\mathrm{E}$

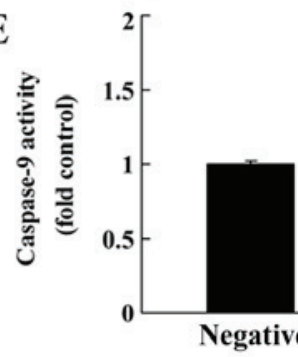

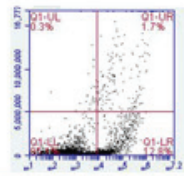

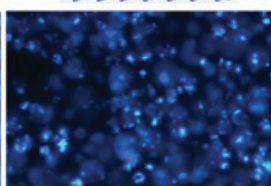

$1 \mathrm{mM}$ Puerarin

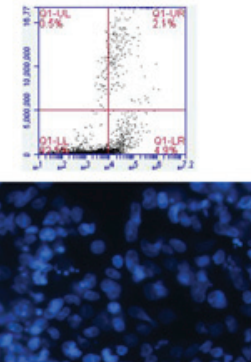

PI3K

Figure 6. Transfection with PI3K plasmids reduces the effect of Puerarin on the apoptosis rate in HeLa cells. (A) Apoptosis rate was determined by (B) flow cytometry. (C) A DAPI assay was performed (magnification, x100). (D) Caspase-3 and (E) caspase-9 activity were assessed. ${ }^{* *} \mathrm{P}<0.01$ vs. the control group. ${ }^{\# \#} \mathrm{P}<0.01$ vs. the $1 \mathrm{nM}$ Puerarin group. PI3K, phosphatidylinositol-3 kinase.

curable (15). In the present study, it was revealed that Puerarin inhibited cell proliferation, increased the LDH activity, reduced the migration rate and increased apoptosis rate in HeLa cells in a dose- and time-dependent manner. A MTT assay, an LDH activity assay and Annexin V-FITC/PI analysis were utilized to analyze cell growth and apoptosis. Hu et al (16) reported that Puerarin inhibits non-small cell lung cancer cell growth via apoptosis induction. However, in future studies it is recommended that the number of experimental methods be expanded to include Ki-67 and Cyclin D analysis. As the current study was only performed with HeLa cells further investigations are required to validate the reported findings.

It has been demonstrated that the risk of developing cervical cancer is positively correlated with the number of sexual partners an individual has, as this increases their exposure to the HPV virus. Smoking, economic status, race and geographical location are all additional risk factors for the development of cervical cancer (17). Gan and Yin (14) demonstrated that Puerarin induced apoptosis in mantle cell lymphoma; based on this, the authors of the present study hypothesized that Puerarin may increase the apoptosis rate of HPV-18 positive cervical cancer cells, which was confirmed in the present study.

The function of $\mathrm{Bcl}-2$ regulates programmed cell death (18). Members of the Bcl-2 family are inhibitors of apoptosis (19). Initiator and executor caspases are key molecules associated with regulation of the apoptosis signaling cascade (19). Apoptosis may occur through 
either the intrinsic or extrinsic signaling pathway (18). The extrinsic pathway is activated by signals from other cells, while the intrinsic pathway may be activated by a number of internal cell signals associated with cell stress, caused by radioactive rays, cytotoxic drugs, the elimination of growth factors and proteins released by mitochondrial membranes, including cytochrome $\mathrm{C}(20)$. The intrinsic pathway is often referred to as the mitochondrial apoptosis-signaling pathway. Cytochrome $\mathrm{C}$ combines with apoptosis protease activating factor 1 and inactivated caspase-9 to form a protein complex called the apoptosome (21). The formation of apoptosomes activates Caspase-9, which in turn activates of a series of caspase proteins (caspase-3, caspase-6 and caspase-7) to trigger changes in cell morphology and biochemistry associated with apoptosis $(22,23)$. The results of the present study indicate that Puerarin effectively induced Bax protein expression and promoted apoptosis in HPV-18 positive cervical cancer cells. Liu et al (24) revealed that Puerarin also suppressed lipopolysaccharide-induced breast cancer cell migration, migration and adhesion.

Activation of the PI3K/mTOR signaling pathway may promote cell cycle progression, reduce apoptosis and promote the migration of cancer cells (23), which are factors associated with the occurrence of multiple tumors (10). Activated PI3K activates downstream Akt (23), which infers an increased tolerance against apoptosis in cancer cells, as well as inducing cell growth and abnormal metabolism (24). The excessive activation of Akt activates downstream mTOR, which may cause the rapid proliferation of cancer cells, increase oncoprotein secretion, accelerate cell cycle progression and shorten the G1 time interval, which increases the occurrence and development of tumors (24). In vivo, mTOR realizes its physiological effects by phosphorylating multiple substrate proteins (24). The translation products include translation elements, such as ribosomal proteins and elongation factors (24). Previous research indicates that mTOR phosphorylates the 412th threonine residue of P70S6 kinase, which increases its activity 100 -fold and promotes the biosynthesis of proteins (25). The results of the present study indicate that Puerarin significantly suppressed PI3K, p-Akt and p-mTOR protein expression in HeLa HPV-18 positive cervical cancer cells, thereby reducing their proliferative and migration abilities; however, PI3K upregulation reduced the anticancer effect of Puerarin on HeLa cells. Huang et al (13) revealed that Puerarin induces cell apoptosis in human chondrosarcoma cells via inhibition of the PI3K/Akt signaling pathway, which supports the results of the present study.

In conclusion, the present study demonstrated that Puerarin effectively inhibits cell proliferation, increases apoptosis and promotes caspase-3/9 and Bax protein expression in HeLa cells, in part by inhibiting the PI3K/Akt/mTOR signaling pathway. However, further studies are required to provide additional evidence for the anticancer effects and underlying mechanisms of Puerarin in HPV-18 positive cervical cancer cells. Puerarin has potential as a novel drug for the treatment of cervical cancer in future clinical practice.

\section{Acknowledgements}

Not applicable.

\section{Funding}

The present study was supported by the National Natural Science Foundation (grant no. 81302538).

\section{Availability of data and materials}

The datasets used and/or analyzed during the current study are available from the corresponding author on reasonable request.

\section{Authors' contributions}

PL designed the experiments. LJ, YH and GY performed the experiments and analyzed the data. PL wrote the manuscript. All authors read and approved the final version of the manuscript.

\section{Ethics approval and consent to participate}

Not applicable.

\section{Patient consent for publication}

Not applicable.

\section{Competing interests}

The authors declare that they have no competing interests.

\section{References}

1. Ruifeng G, Yunhe F, Zhengkai W, Ershun Z, Yimeng L, Minjun Y, Xiaojing S, Zhengtao Y and Naisheng Z: Chlorogenic acid attenuates lipopolysaccharide-induced mice mastitis by suppressing TLR4-mediated NF-kappaB signaling pathway. Eur J Pharmacol 729: 54-58, 2014.

2. Livak KJ and Schmittgen TD: Analysis of relative gene expression data using real-time quantitative PCR and the 2(-Delta Delta C(T)) method. Methods 25: 402-408, 2001.

3. Jiang H, Li J, Chen A, Li Y, Xia M, Guo P, Yao S and Chen S: Fucosterol exhibits selective antitumor anticancer activity against HeLa human cervical cell line by inducing mitochondrial mediated apoptosis, cell cycle migration inhibition and downregulation of m-TOR/PI3K/Akt signalling pathway. Oncol Lett 15: 3458-3463, 2018.

4. Kemeny N, Brown K, Covey A, Kim T, Bhargava A, Brody L, Guilfoyle B, Haag NP, Karrasch M, Glasschroeder B, et al: Phase I, open-label, dose-escalating study of a genetically engineered herpes simplex virus, NV1020, in subjects with metastatic colorectal carcinoma to the liver. Hum Gene Ther 17: 1214-1224, 2006.

5. LiCH, Shi L, Zhan GL, Rao SZ and Zhang H: A twenty-four-week, open-label study on ziprasidone's efficacy and influence on glucolipid metabolism in patients with schizophrenia and metabolic disorder. Eur Rev Med Pharmacol Sci 17: 2136-2140, 2013.

6. Bettencourt C, Santos C, Montiel R, Kay T, Vasconcelos J, Maciel P and Lima M: The (CAG)n tract of Machado-Joseph Disease gene (ATXN3): A comparison between DNA and mRNA in patients and controls. Eur J Hum Genet 18: 621-623, 2010.

7. Chang KH, Chen WL, Wu YR, Lin TH, Wu YC, Chao CY, Lin JY, Lee LC, Chen YC, Lee-Chen GJ and Chen CM: Aqueous extract of Gardenia jasminoides targeting oxidative stress to reduce polyQ aggregation in cell models of spinocerebellar ataxia 3. Neuropharmacology 81: 166-175, 2014.

8. Mukohyama J, Shimono Y, Minami H, Kakeji Y and Suzuki A: Roles of microRNAs and RNA-binding proteins in the regulation of colorectal cancer stem cells. Cancers (Basel) 9: E143, 2017.

9. Zhang B, Tian M, Zhen Y, Yue Y, Sherman J, Zheng H, Li S, Tanzi RE, Marcantonio ER and Xie Z: The effects of isoflurane and desflurane on cognitive function in humans. Anesth Analg 114: 410-415, 2012. 
10. Li H, Yao C, Shi J, Yang F, Qi S, Wang L, Zhang H, Li J, Wang C, Wang C, et al: Comparative study of the efficacy and safety between blonanserin and risperidone for the treatment of schizophrenia in Chinese patients: A double-blind, parallel-group multicenter randomized trial. J Psychiatr Res 69: 102-109, 2015.

11. Haacke A, Broadley SA, Boteva R, Tzvetkov N, Hartl FU and Breuer P: Proteolytic cleavage of polyglutamine-expanded ataxin-3 is critical for aggregation and sequestration of non-expanded ataxin-3. Hum Mol Genet 15: 555-568, 2006.

12. Chen $\mathrm{L}$ and Madura K: Evidence for distinct functions for human DNA repair factors hHR23A and hHR23B. FEBS Lett 580: 3401-3408, 2006.

13. Huang L, Cao J, Cao L, Gao L, Yang Y and Xu L: Puerarin induces cell apoptosis in human chondrosarcoma cell line SW1353 via inhibition of the PI3K/Akt signaling pathway. Oncol Lett 14: 5585-5590, 2017.

14. Gan M and Yin X: Puerarin induced in mantle cell lymphoma apoptosis and its possible mechanisms involving multi-signaling pathway. Cell Biochem Biophys 71: 367-373, 2015.

15. Sun HQ, Li SX, Chen FB, Zhang Y, Li P, Jin M, Sun Y, Wang F, Mi WF, Shi L, et al: Diurnal neurobiological alterations after exposure to clozapine in first-episode schizophrenia patients. Psychoneuroendocrinology 64: 108-116, 2016.

16. Hu Y, Li X, Lin L, Liang S and Yan J: Puerarin inhibits non-small cell lung cancer cell growth via the induction of apoptosis. Oncol Rep 39: 1731-1738, 2018.

17. Cascinu S, Rosati G, Nasti G, Lonardi S, Zaniboni A, Marchetti P, Leone F, Bilancia D, Iaffaioli RV, Zagonel V, et al: Treatment sequence with either irinotecan/cetuximab followed by FOLFOX-4 or the reverse strategy in metastatic colorectal cancer patients progressing after first-line FOLFIRI/bevacizumab: An Italian Group for the Study of Gastrointestinal Cancer phase III, randomised trial comparing two sequences of therapy in colorectal metastatic patients. Eur J Cancer 83: 106-115, 2017.
18. Chen L, Zhang B, Shan S and Zhao X: Neuroprotective effects of vitexin against isoflurane-induced neurotoxicity by targeting the TRPV1 and NR2B signaling pathways. Mol Med Rep 14: 5607-5613, 2016

19. Chang M, Sun L, Liu X, Sun W and You X: Association of common variants in H2AFZ gene with schizophrenia and cognitive function in patients with schizophrenia. J Hum Genet 60: 619-624, 2015.

20. Li G, Xue Q, Luo Y, Hu X and Yu B: S6 inhibition contributes to isoflurane neurotoxicity in the developing brain. Toxicol Lett 233: 102-113, 2015

21. Chai D, Jiang H and Li Q: Isoflurane neurotoxicity involves activation of hypoxia inducible factor-1 $\alpha$ via intracellular calcium in neonatal rodents. Brain Res 1653: 39-50, 2016.

22. Scurr M, Pembroke T, Bloom A, Roberts D, Thomson A, Smart K, Bridgeman H, Adams R, Brewster A, Jones R, et al: Effect of modified vaccinia ankara-5T4 and low-dose cyclophosphamide on antitumor immunity in metastatic colorectal cancer: A randomized clinical trial. JAMA Oncol 3: e172579, 2017.

23. Man DW, Law KM and Chung RC: Cognitive training for Hong Kong Chinese with schizophrenia in vocational rehabilitation. Hong Kong Med J 18 (Suppl 6): S18-S22, 2012.

24. Liu F, Guo X, Wu R, Ou J, Zheng Y, Zhang B, Xie L, Zhang L, Yang L, Yang S, et al: Minocycline supplementation for treatment of negative symptoms in early-phase schizophrenia: A double blind, randomized, controlled trial. Schizophr Res 153: 169-176, 2014.

25. Lane HY, Liu YC, Huang CL, Chang YC, Liau CH, Perng CH and Tsai GE: Sarcosine (N-methylglycine) treatment for acute schizophrenia: A randomized, double-blind study. Biol Psychiatry 63: 9-12, 2008.

This work is licensed under a Creative Commons Attribution-NonCommercial-NoDerivatives 4.0 International (CC BY-NC-ND 4.0) License. 